\title{
Coinfecção fusariose e criptococose em paciente imunocomprometida: relato de caso
}

\author{
Fusariosis and cryptococcosis co-infection in an immunocompromised patient: case report
}

Coinfección fusariosis y criptococcosis en una paciente inmunocomprometida: caso clínico

Tayrel dos Anjos Silva ${ }^{1 *}$, Rejane Nina Martins ${ }^{1}$, Luiz Felipe Campos ${ }^{2}$, Adriano de Aquino da Luz ${ }^{3}$, Natasha Valois Castelo Branco ${ }^{1}$, Francisco Marcelo Saraiva Luna1.

\section{RESUMO}

Objetivo: Relatar caso de uma paciente imunossuprimida, internada em enfermaria, evoluindo com coinfecção por fusariose e criptococose devido a aplasia após quimioterapia para tratamento de leucemia mielóide aguda (LMA) refratária. Detalhamento de caso: Mulher de 24 anos, previamente hígida, teve diagnóstico de LMA em abril de 2018, foi submetida a quimioterapia em duas ocasiões, a primeira com citarabina e idarrubicina com resposta parcial e remissão incompleta, sendo sucedido do protocolo com as mesmas drogas em altas doses, apresentando remissão completa eventual. Em novembro de 2018, foi diagnosticada recidiva da doença, apresentando lesões nodulares eritemato-violáceas, sendo confirmada fusariose com estudo anatomopatológico e hemocultura para fungos positiva para Cryptococcus laurentii. Realizado tratamento com voriconazol e anfotericina B desoxicolato, obteve pouca resposta. Com a falha terapêutica, foi instituído protocolo de cuidados paliativos, evoluindo com óbito no décimo quinto dia de internação. Considerações finais: A aplasia após infusão da quimioterapia é esperada por volta do décimo dia, sendo que a queda dos glóbulos brancos é um fenômeno preocupante, podendo ser fatal. O estado de neutropenia severa pode deixar o paciente susceptível a infecções, impondo um estado de alerta constante à equipe de saúde assistente.

Palavras-chave: Leucemia mieloide aguda, Fusariose, Criptococose.

\section{ABSTRACT}

Objective: To report a case of an immunosuppressed patient, admitted to the ward, who developed fusariosis and cryptococcosis coinfection due to aplasia after chemotherapy for the treatment of refractory acute myeloid leukemia (AML). Case details: A 24 year old woman, previously healthy, was diagnosed with AML in April 2018, underwent chemotherapy on two occasions, the first with cytarabine and idarubicin with partial response and incomplete remission, followed by the protocol with the same drugs in high doses, showing eventual complete remission. In November 2018, disease recurrence was diagnosed, with erythematous-violaceous nodular lesions, and fusariosis was confirmed with anatomopathological study and positive fungal blood culture for Cryptococcus laurentii. After treatment with voriconazole and amphotericin B deoxycholate, there was little response. With therapeutic failure, a palliative care protocol was instituted, evolving with death on the fifteenth day of hospitalization. Final considerations: Aplasia after chemotherapy infusion is expected around the tenth day, and the fall of white blood cells is a worrying phenomenon, which can be fatal. The state of severe neutropenia can leave the patient susceptible to infections, imposing a constant state of alert on the assistant health team.

Key words: Acute myeloid leukemia, Fusariosis, Cryptococcosis.

1Universidade Federal do Amazonas (UFAM), Manaus - AM. *E-mail: tayreldosanjos@hotmail.com

${ }^{2}$ Fundação de Medicina Tropical Doutor Heitor Vieira Dourado, Manaus - AM.

${ }^{3}$ Secretaria de Estado de Saúde do Amazonas, Manaus - AM.

SUBMETIDO EM: 5/2020

ACEITO EM: 6/2020

PUBLICADO EM: 7/2020 


\section{RESUMEN}

Objetivo: Relatar caso de una paciente inmunodeprimida, admitida en la enfermería, que desarrolló coinfección por fusariosis y criptococosis debido a aplasia después de la quimioterapia para el tratamiento de leucemia mieloide aguda (LMA) refractaria. Detalles del caso: Mujer de 24 años, previamente sana, fue diagnosticada con LMA en abril de 2018, recibió quimioterapia en dos ocasiones, la primera con citarabina e idarubicina con respuesta parcial y remisión incompleta, seguido de nuevo protocolo con los mismos medicamentos en altas dosis, mostrando remisión completa eventual. En noviembre de 2018, se diagnosticó la recurrencia de la enfermedad, con lesiones nodulares eritematosas-violáceas, que se confirmó fusariosis en estudio anatomopatológico y hemocultivo para hongos positivo para Cryptococcus laurentii. Después del tratamiento con voriconazol y anfotericina B desoxicolato, hubo poca respuesta. Con el fracaso terapéutico, se instituyó un protocolo de cuidados paliativos, que evolucionó con la muerte al decimoquinto día de hospitalización. Consideraciones finales: Esperase aplasia después de la infusión de quimioterapia alrededor del décimo día, y la caída de glóbulos blancos es un fenómeno preocupante, que puede ser fatal. El estado de neutropenia severa puede dejar al paciente susceptible a infecciones, imponiendo un estado de alerta constante en el equipo auxiliar de salud.

Palabras clave: Leucemia mieloide aguda, Fusariosis, Criptococosis.

\section{INTRODUÇÃO}

A incidência de câncer cresce anualmente em todo o mundo, sendo que, a estimativa para o ano de 2020 é de 10810 novos casos no Brasil (INCA, 2020). Na literatura são raros os relatos de casos sobre coinfecção por criptococose e fusariose, mesmo em indivíduos com patologias imunossupressoras amplamente estudadas, como nas leucemias.

As leucemias agudas são neoplasias decorrentes de transformação maligna das células hematopoiéticas primitivas, seguida de uma proliferação clonal e seu consequente acúmulo no organismo. A Leucemia Mieloide Aguda caracteriza-se por uma parada da maturação celular na fase de blastos ou promielócitos que leva à redução dos elementos normais no sangue periférico. Seu tratamento é composto por quimioterapia de indução, com o objetivo de atingir remissão completa da doença e consequente restauração das células sanguíneas normais. Esta fase é seguida por uma terapia de pós-remissão para erradicar a doença residual mínima (DRM). Por conseguinte, dois a quatro cursos de consolidação com ou sem tratamento prolongado de manutenção (MINISTÉRIO DA SAÚDE, 2014).

Ainda de acordo com o Ministério da Saúde (2014), independentemente da idade, o objetivo inicial do tratamento da LMA é reduzir a celularidade da medula óssea para menos de $5 \%$ de mieloblastos e, no sangue periférico, neutrófilos acima de $1.000 / \mathrm{mm}^{3}$ e plaquetas acima de $100.000 / \mathrm{mm}^{3}$. Considera-se indivíduo potencialmente curado, aqueles que mantém o estado de remissão durante dois a três anos, período este que o risco de recaída é inferior a 10\%. Uma das principais complicações do paciente leucêmico é a imunossupressão decorrente da própria doença ou induzida pela quimioterapia.

As infecções fúngicas sistêmicas são as principais causas de morbimortalidade em pacientes com neoplasias hematológicas. Embora outras espécies fúngicas possam acometer pacientes imunossuprimidos, as infecções oportunistas mais prevalentes são: a candidíase, por Candida albicans e outras espécies; a aspergilose, principalmente por Aspergillus fumigatus; a criptococose por Cryptococcus neoformans e as mucormicoses (NAKAMURA HM, et al., 2013).

A fusariose invasiva é uma doença fúngica grave que afeta principalmente pacientes com neoplasias hematológicas e receptores de transplante de células hematopoiéticas (NUCCI M, 2007). As manifestações clínicas mais observadas em indivíduos imunossuprimidos são: febre persistente, refratária à terapia antibacteriana e antifúngica, sinusite, pneumonia, celulite e endoftalmite (ANAISSIE e NUCCI M, et al., 2019). Essa correlação pode ser observada em um estudo realizado no Hospital Universitário da Universidade Federal do Rio de Janeiro, o qual concluiu que pacientes com diagnóstico de neoplasias hematológicas acometidos por lesões cutâneas apresentavam maior risco de desenvolver sua forma invasiva, principalmente durante estado de neutropenia (VARON AG, et al., 2014). 
As espécies de Fusarium são tradicionalmente estudadas como importante patógeno de plantas por causarem doenças como podridão da coroa, murcha de Fusarium e cabeça de ferrugem (NELSON PE, 1994). Em humanos, podem causar infecções superficiais como ceratite e onicomicose, localmente invasivas ou disseminadas (NUCCI M e ANAISSIE E, 2007). É uma complicação importante para pacientes imunossuprimidos e estão associadas à alta morbimortalidade (NUCCI M, 2002).

Três espécies são clinicamente importantes: F. solani, F. oxysporum e F. verticillioidis. Tais fungos são considerados desafios terapêuticos, por serem resistentes a maioria dos antifúngicos disponíveis, restringindo de maneira significativa os recursos terapêuticos (SIFUENTES-OSORNIO, 2012).

A criptococose é uma doença fúngica, causada por dois agentes etiológicos: Cryptococcus neoformans e Cryptococcus gattii, pertencentes ao filo Basidiomycota que podem ser facilmente distinguidos das outras leveduras patogênicas, como Candida, pela presença de uma cápsula polissacarídica, formação de melanina e atividade da urease, que funcionam como determinantes da virulência. A infecção é transmitida por inalação e subsequente disseminação para outros sistemas (KWON-CHUNG KJ, et al., 2014).

As espécies de Cryptococcus não neoformans, incluindo $C$. laurentii e $C$. albidus, historicamente consideradas saprófitas e não patogênicas tem sido associadas, nas últimas décadas, a infecções oportunistas, com acometimento de pele, pulmões, corrente sanguínea e sistema nervoso central (SNC) em pacientes imunocomprometidos (SMITH M, et al., 2017). Cryptococcus laurentii e Cryptococcus albidus, juntos, são responsáveis por aproximadamente $80 \%$ dos casos relatados (KHAWCHAROENPORN T et al., 2007).

Dentre os fatores de risco associados à infecção por $C$. laurentii, o mais comumente observados são: dispositivos invasivos, exposição prévia a esteroides, exposição prévia a imunossupressores, exposição prévia a derivados azólicos, baixa contagem de CD4, exposição a excrementos de pombos e neutropenia (KHAWCHAROENPORN T, et al., 2007).

Os criptococos são intrinsecamente resistentes às equinocandinas, tendo, portanto, seu manejo clínico mais difícil, apresentando a ocorrência de frequentes efeitos adversos decorrentes da terapia medicamentosa. O tratamento ideal para infecções invasivas devido às espécies menos comuns de Cryptococcus não foi estabelecido, podendo ser empregadas anfotericina B isolada ou em combinação com fluconazol (ARENDRUP MC, 2014).

Este estudo tem por objetivo, relatar o caso de uma paciente imunocomprometida, internada em enfermaria, evoluindo com coinfecção por fusariose e criptococose devido à aplasia após quimioterapia para tratamento de leucemia mielóide aguda refratária.

\section{DETALHAMENTO DO CASO}

Paciente do sexo feminino, 24 anos, previamente hígida, durante investigação de leucocitose, teve diagnóstico de leucemia mielóide aguda em abril de 2018, sendo na ocasião, submetida a dois protocolos de quimioterapia com objetivo de remissão da doença. O primeiro foi realizado com associação de citarabina e idarrubicina no período de março a maio de 2018, com resposta parcial e remissão incompleta da doença, sendo necessário realizar o protocolo com as mesmas drogas em altas doses, no período de julho a agosto, apresentando remissão completa eventual e boa resposta ao tratamento. O segundo protocolo foi iniciado a partir do diagnóstico de recidiva da doença em novembro de 2018, com o emprego da terapia com citarabina, idarrubicina e fludarabina (IDA-FLAG) e filgrastima, apresentando resposta insatisfatória.

No dia 26 de dezembro de 2018 em consulta ambulatorial de rotina, apesar da ausência de queixas clínicas, possuía mielograma com resultado hipercelular para a idade, com hiperplasia de eritroblastos e $10 \%$ de blastos no sangue periférico. Após o segundo ciclo de IDA-FLAG evoluiu com piora do leucograma, sendo indicada internação hospitalar em janeiro de 2019 para realizar o terceiro ciclo quimioterápico, como nova tentativa de se obter aplasia de medula.

Ao exame físico da admissão, foram observadas escoriações em joelho direito, petéquias disseminadas pelo tronco, membros superiores e inferiores e a presença de um nódulo arredondado, eritemato-violáceo, de aproximadamente um centímetro de diâmetro, localizado no antebraço direito. No decorrer da internação 
hospitalar evoluiu com êmeses recorrentes, odinofagia, câimbras e paraparestesia de membros inferiores, além de dor torácica ventilatória-dependente. Ao estudo radiográfico do tórax não foram observadas alterações, o ecocardiograma com doppler evidenciou cavidade esquerda com dimensões limítrofes, derrame pericárdico mínimo, derrame pleural bilateral leve e ascite, dois testes de escarro apresentaram resultado negativo para tuberculose e dois hemogramas com alterações compatíveis com a neoplasia hematológica de base e aplasia após quimioterapia.

A partir da segunda semana de internação em isolamento, a paciente evoluiu com lesões nodulares eritemato-violáceas, inicialmente em membros superiores (Figura 1), tronco e face, posteriormente com acometimento de todo o corpo, as quais tornaram-se levemente ulceradas e dolorosas, frequentemente associadas à picos febris que não cediam ao uso do antipiréticos. Foi realizado estudo anatomopatológico de algumas lesões descritas, cujo resultado foi de infecção por Fusarium.

Figura 1 - Lesões nodulares eritemato-violáceas.

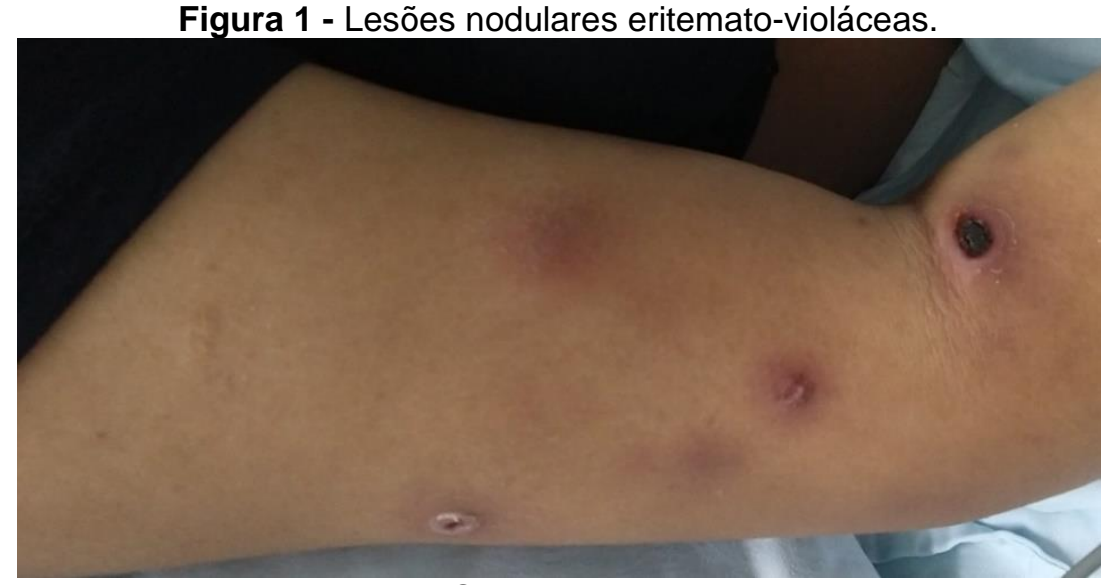

Fonte: Silva TA, et al., 2019.

A paciente já fazia uso profilático de Sufametoxazol com Trimetoprima devido a linfopenia importante, passou a receber tratamento antimicrobiano com meropenem, vancomicina e Anfotericina B desoxicolato. Ainda com resposta satisfatória, no dia 12 de fevereiro foi recebido o resultado de hemocultura positiva para Cryptococcus laurentii, fez-se necessário o ajuste da terapia com a prescrição de voriconazol em associação a anfotericina $B$ desoxicolato. Apesar de receber cobertura antimicrobiana otimizada para as duas infecções fúngicas, não houve melhora do quadro geral da paciente, a qual evoluiu com desconforto respiratório progressivo refratário.

Dessa forma, após a reunião familiar, procederam-se às medidas de cuidados paliativos exclusivos em consonância com a conduta compartilhada entre a equipe multiprofissional, a família e a paciente. Foi ofertada terapia com sedação e analgesia em infusão contínua, ajustada conforme necessidade da paciente, monitorização contínua para estabelecer parâmetros objetivos quanto ao conforto e com auxílio da equipe de fisioterapia, foi ofertado suporte ventilatório não invasivo ajustado conforme evolução do desconforto ventilatório. Seguindo o protocolo dos cuidados paliativos estabelecidos na reunião familiar, a paciente evoluiu com óbito no dia 18 de fevereiro de 2019.

\section{DISCUSSÃO}

Nas plataformas pesquisadas, não foram encontrados relatos de casos sobre coinfecção por criptococose e fusariose, apesar de serem patologias comuns e continuamente estudadas, principalmente em grupos de pacientes imunossuprimidos como leucemias e síndrome da imunodeficiência adquirida. De acordo com Miranda BA (2018), em sua revisão sistemática, a criptococose manifesta-se em coinfecção com diversos outros patógenos, como candidíase, tuberculose e não aborda no estudo a associação com fusariose.

Em estudo retrospectivo multicêntrico, Nucci M, et al. (2019), concluíram que os pacientes que necessitavam de curso adicional de quimioterapia ou terapia imunossupressora se beneficiavam de profilaxia secundária para criptococose, principalmente nos casos de doença disseminada. Desse modo, observa-se 
que pacientes com leucemias são especialmente acometidos por doenças fúngicas oportunistas, inerentes ao tratamento da doença de base, com imunossupressão após quimioterapia, fazendo-se necessários cuidados específicos de isolamento e profilaxias primárias e secundárias. A aplasia após infusão da quimioterapia é esperada por volta do décimo dia, sendo que a queda dos glóbulos brancos é um fenômeno preocupante, podendo ser fatal. Como também, observa-se a necessidade de medidas profiláticas, com terapias farmacológicas e não farmacológicas, conforme a necessidade de cada caso.

Estudo realizado no Hospital das Clínicas da Universidade de Campinas demostrou, pela primeira vez, uma relação genética entre espécies de Fusarium isoladas do ar interno de hospitais com aquelas recuperadas em hemoculturas de pacientes hematológicos, sugerindo que o ar pode ser uma fonte potencial de fusariose (MORETTI ML, et al., 2018). Tal avaliação é importante pois os indivíduos vulneráveis são infectados com fungos cujos esporos estão presentes no ar ambiente e com base nessa informação é possível assumir medidas para prevenir essas infecções.

Segundo Khawcharoenporn T, et al. (2007), pacientes com dispositivos invasivos, exposição prévia a esteroides, imunossupressores ou derivados azólicos, baixa contagem de CD4, exposição a excrementos de pombos e neutropenia são mais susceptíveis a infecção fúngica invasiva, dentre elas a criptococose e a fusariose. No caso descrito, a paciente possuía uma doença neoplásica hematológica, que por sua vez já levava a um estado de imunossupressão pela infiltração da medula óssea com células neoplásicas, tinha como tratamento inicial a quimioterapia cujo efeito final visava a aplasia medular. Assim, tanto a doença de base quando o tratamento eram fatores imunossupressores que facilitavam o acometimento de infecções oportunistas.

Outrossim, pacientes oncológicos apresentam grande sofrimento psicológico durante todo o processo de adoecimento, desde os primeiros sintomas, investigação diagnóstica, submissão aos exames muitas vezes invasivos, antes, durante e após o tratamento instituído e na reabilitação. Diante disso, é fundamental haver suporte de equipe multidisciplinar para assistência mais humanizada. Segundo Boucher NA, et al. (2019), este tipo de sofrimento muitas vezes é esquecido, apesar de serem comuns em populações com neoplasias, ressaltando em seu estudo que muitos indivíduos observados tiveram o tempo de sofrimento prolongado além do esperado com o tratamento, evidenciando que a resolução ou controle da doença física não é único determinante para conforto psicológico.

Associado ao tratamento farmacológico, a atuação da fisioterapia é fundamental para melhora da qualidade de vida durante os cuidados paliativos. Segundo Cipolat S, et al. (2011), os pacientes imunossuprimidos permanecem por um longo período em isolamento e ficam expostos a uma diversidade de toxinas quimioterápicas, às quais restringem as atividades físicas e potencializam os efeitos deletérios sobre o sistema cardiopulmonar. A alta morbimortalidade resultante tanto do processo de infiltração neoplásica quanto da infecção disseminada, instiga a atuação fisioterapêutica na prevenção de complicações como pneumonias intersticiais e infecciosas.

Portanto, a fisioterapia pode auxiliar no tratamento melhorando a função global ou auxiliando na sintomatologia, através de incentivadores respiratórios como a ventilação não invasiva, técnicas de expansão pulmonar que promovem uma maior contração dos músculos intercostais e do diafragma, estimulando um maior esforço inspiratório. Portanto, necessita-se também de trabalho de alongamento, fortalecimento muscular, orientações posturais para prevenir as algias musculares decorrentes da imobilização.

No protocolo de atendimento da paciente em questão, foram realizadas como medidas de conforto no processo de fim de vida, o suporte ventilatório não invasivo, a fim de reduzir o desconforto respiratório decorrente da fraqueza muscular generalizada, dos processos infecciosos e infiltrativos resultantes da evolução do quadro clínico, mediante falha do tratamento instituído.

A aplasia após infusão da quimioterapia é esperada por volta do décimo dia, sendo que a queda dos glóbulos brancos pode levar a um estado de imunossupressão que pode ser fatal. A neutropenia severa pode deixar o paciente susceptível a infecções, impondo um estado de alerta constante à equipe de saúde assistente. Após realização de busca em plataformas de pesquisa científica, não foram encontrados casos 
relatados sobre coinfecção por fusariose e criptococose, apesar de, individualmente, serem infecções fúngicas que normalmente acometem pacientes imunodeprimidos. Portanto, falta maior publicidade dos casos em que as duas infeções acometam simultaneamente, na forma invasiva, o mesmo indivíduo. Assim, esperase que este relato contribua para publicações de novos estudos sobre o tema.

\section{REFERÊNCIAS}

1. ANAISSIE E, NUCCI M. Clinical manifestations and diagnosis of Fusarium infection. Uptodate. 2019.

2. ARENDRUP MC. ESCMID and ECMM joint clinical guidelines for the diagnosis and management of rare invasive yeast infections. Clin Microbiol Infect. 2014; 20(3): 76-98.

3. BOUCHER NA, et al. Acute Leukemia Patients' Needs: Qualitative Findings and Opportunities for Early Palliative Care. Journal of Pain and Symptom Management, 2018; 55(2): 433-439.

4. CIPOLAT S, et al. Fisioterapia em pacientes com leucemia : Revisão Sistemática. Revista Brasileira de Cancerologia. 2011; 57(2): 229-236.

5. INSTITUTO NACIONAL DE CÂNCER - INCA. Estimativa 2020 : Incidência de câncer no Brasil. Rio de Janeiro. 2020.

6. KHAWCHAROENPORN T, et al. Non-neoformans Cryptococcal Infections: a Systematic Review. Infection. 2007; 35(2): 51-8.

7. KWON-CHUNG KJ, et al. Cryptococcus neoformans and Cryptococcus gattii, the etiologic agents of cryptococcosis. Cold Spring Harb Perspect Med. 2014; 4(7): a019760.

8. MINISTÉRIO DA SAÚDE - Secretaria de Atenção à Saúde. Protocolos Clínicos e Diretrizes Terapêuticas em Oncologia. Brasília. 2014.

9. MIRANDA BA. Coinfecções no contexto da criptococose: levantamento de estudos clínicos e experimentais, MG. Monografia (Especialização em Microbiologia) - Instituto de Ciências Biológicas. Universidade Federal de Minas Gerais, Belo Horizonte, 2018; 70p.

10. MORETTI ML, et al. Airborne transmission of invasive fusariosis in patients with hematologic malignancies. PLoS ONE. 2018; 13(4): e0196426.

11. NAKAMURA HM, et al. Incidência de infecções fúngicas em pacientes cirúrgicos: uma abordagem retrospectiva. Rev. SOBECC. 2013; 18(3): 49-58.

12. NELSON PE, et al. Taxonomy, Biology, and Clinical Aspects of Fusarium Species. Clinical Microbiology Reviews. 1994; 7(4): 479-504.

13. NUCCI M. Cutaneous Infection by Fusarium Species in Healthy and Immunocompromised Hosts: Implications for Diagnosis and Management. Clinical Infectious Diseases, 2002, 35(8): 909-920.

14. NUCCI M, ANAISSIE E. Fusarium Infections in Immunocompromised Patients. Clinical Microbiology Reviews, 2007; 20(4): 695-704.

15. NUCCI M, et al. Outcomes of patients with invasive fusariosis who undergo further immunosuppressive treatments, is there a role for secondary prophylaxis?. Mycoses, 2019; 62(5): 413-417.

16. SIFUENTES-OSORNIO J, et al. Epidemiology of Invasive Fungal Infections in Latin America. Current Fungal Infection Reports. 2012; 6, 23-34.

17. SMITH N, et al. Perspectives on non-neoformans cryptococcal opportunistic infections. J Community Hosp Intern Med Perspect, 2017; 7(4):214-217.

18. VARON AG, et al. Superficial skin lesions positive for Fusarium are associated with subsequent development of invasive fusariosis. J. Infect. 2014; 68, 85-89. 